\title{
Strain-Rate Frequency Superposition: A Rheological Probe of Structural Relaxation in Soft Materials
}

\author{
Hans M. Wyss, ${ }^{1}$ Kunimasa Miyazaki, ${ }^{2,3}$ Johan Mattsson, ${ }^{1}$ Zhibing Hu, ${ }^{4}$ David R. Reichman, ${ }^{2}$ and David A. Weitz ${ }^{1}$ \\ ${ }^{1}$ Department of Physics \& HSEAS, Harvard University, Cambridge, Massachusetts 02138, USA \\ ${ }^{2}$ Department of Chemistry, Columbia University, New York, New York 10027, USA \\ ${ }^{3}$ The Research Institute of Kochi University of Technology, Tosa Yamada, Kochi 782-8502, Japan \\ ${ }^{4}$ Department of Physics, University of North Texas, Denton, Texas 76203, USA
}

(Received 18 August 2006; published 7 June 2007)

\begin{abstract}
The rheological properties of soft materials often exhibit surprisingly universal linear and nonlinear features. Here we show that these properties can be unified by considering the effect of the strain-rate amplitude on the structural relaxation of the material. We present a new form of oscillatory rheology, strain-rate frequency superposition (SRFS), where the strain-rate amplitude is fixed as the frequency is varied. We show that SRFS can isolate the response due to structural relaxation, even when it occurs at frequencies too low to be accessible with standard techniques.
\end{abstract}

DOI: 10.1103/PhysRevLett.98.238303

PACS numbers: $83.60 . \mathrm{Bc}, 83.80 . \mathrm{Hj}, 83.80 . \mathrm{Iz}$

Concentrated suspensions [1], pastes [2], emulsions [3], foams [4], or associative polymer systems [5,6] represent commonly encountered examples of soft solids. Their structures are often metastable, with dynamics strongly reminiscent of that of glasses. They are thus often termed soft glassy materials [7]. Despite the wide diversity of these materials, their rheological response, as characterized by the complex shear modulus $G^{*}(\omega)=G^{\prime}(\omega)+$ $i G^{\prime \prime}(\omega)$, is remarkably similar. The storage modulus $G^{\prime}$ is only very weakly dependent on frequency. The loss modulus $G^{\prime \prime}$ also shows a very weak frequency dependence and is much larger than the response due to the continuous phase fluid. It often exhibits a shallow minimum near the lowest experimentally accessible frequencies. At even lower frequencies, there should be a crossover from solidlike to liquidlike behavior, which is signaled by a pronounced peak in $G^{\prime \prime}(\omega)$, reflecting the structural relaxation. Unfortunately, the relaxation frequencies are often much too low to be accessed by standard linear rheological measurements. A typical example of this linear viscoelastic behavior is shown in Fig. 1(a) for soft hydrogel spheres. Remarkably, the similarities in the rheological response of these materials extend even to nonlinear measurements, characterized by strain-dependent viscoelastic measurements performed at constant $\omega$, varying the strain amplitude. Above a critical yield strain, the storage modulus exhibits a power-law decay $G^{\prime}\left(\gamma_{0}\right) \propto \gamma_{0}^{-\nu^{\prime}}$. By contrast, the loss modulus exhibits a well-defined peak, before falling as a power-law $G^{\prime \prime}\left(\gamma_{0}\right) \propto \gamma_{0}^{-\nu^{\prime \prime}}$; typically with $\nu^{\prime \prime} \approx \nu^{\prime} / 2$. The pronounced peak in the loss modulus is a remarkably robust feature of soft glassy materials $[1,3,5,6,8]$. The ubiquitousness and similarity of the rheological response, both linear and nonlinear, of so many soft materials suggests that the response is governed by a common underlying mechanism. A possible clue to the origin of this behavior comes from a proposed explanation for the response of a supercooled fluid [9]. Within this picture, the peak in $G^{\prime \prime}\left(\gamma_{0}\right)$ observed in nonlinear measurements is directly related to a decrease of the structural relaxation time with increasing shear rate [7,10-13]; the applied strain drives the relaxation and forces it to a higher frequency, where it is directly probed. While this picture provides an excellent description of a colloidal supercooled liquid, the underlying physical concept should be much more generally applicable. If this link is verified, the relaxation could be probed using the nonlinear response, even when the relaxation frequency is experimentally inaccessible. This would provide a new probe of the dynamics and rheology of soft materials. However, there have been no attempts to exploit this behavior and explore its general applicability to a variety of different materials.

In this Letter, we show that the typical linear and nonlinear viscoelastic behavior of a wide variety of soft materials can be explained by a common mechanism [7,10-13]. We introduce a new approach to oscillatory rheology; by keeping the strain rate constant during the measurement we

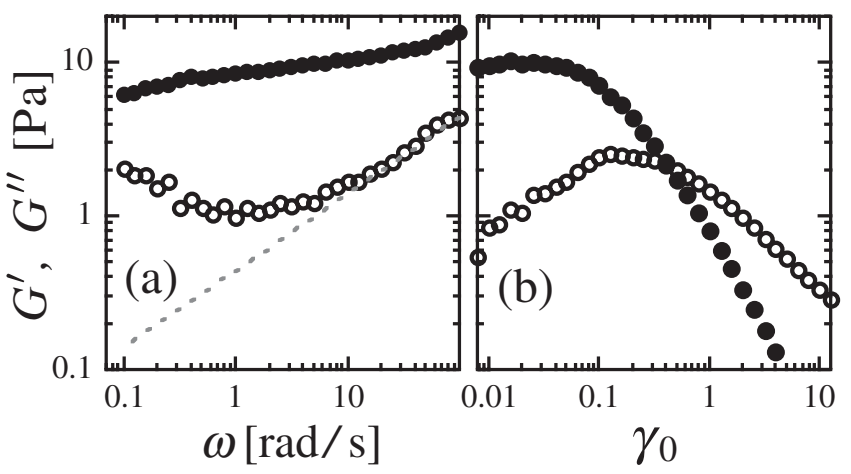

FIG. 1. Oscillatory measurements of $G^{\prime}$ (solid circles) and $G^{\prime \prime}$ (open circles) for a suspension of hydrogel particles. (a) Linear frequency dependent measurement at $\gamma_{0}=0.02$. The dashed line is proportional to $\sqrt{\omega}$. (b) Strain-dependent nonlinear measurement at $\omega=1 \mathrm{rad} / \mathrm{s}$. 
can directly probe the structural response. The data exhibits a characteristic scaling, allowing us to clearly isolate and probe the response due to structural relaxation. This new technique, which we call strain-rate frequency superposition (SRFS), should be generally applicable to the study of many soft materials.

To elucidate the viscoelastic response, we use a system of hydrogel microspheres dispersed in water. They consist of interpenetrating polymer networks of cross-linked poly $N$-isopropylacrylamide and polyacrylic acid $[14,15]$. The sample studied has a polymer weight fraction $c_{p}=0.0186$ and at low volume fraction the particles have a radius of $R \simeq 95 \mathrm{~nm}$. All rheological measurements are performed with a strain-controlled rheometer, applying an oscillatory strain $\gamma(t)$ to the sample and measuring the resultant timedependent stress $\sigma(t)$.

This system of soft spheres shows the behavior typical of soft materials, both in its linear and nonlinear viscoelastic behavior, including the ubiquitous peak in $G^{\prime \prime}\left(\gamma_{0}\right)$ that typefies the rheology. The measurements shown in Fig. 1 represent the most commonly used types of oscillatory rheological measurements. They depend on two variables only, the frequency $\omega$ and the strain amplitude $\gamma_{0}$; the applied oscillatory strain for each data point is $\gamma(t)=$ $\gamma_{0} \sin (\omega t)$. Frequency dependent measurements [Fig. 1(a)] are usually performed in the linear viscoelastic regime at a constant $\gamma_{0} \ll 1$, while strain-dependent measurements

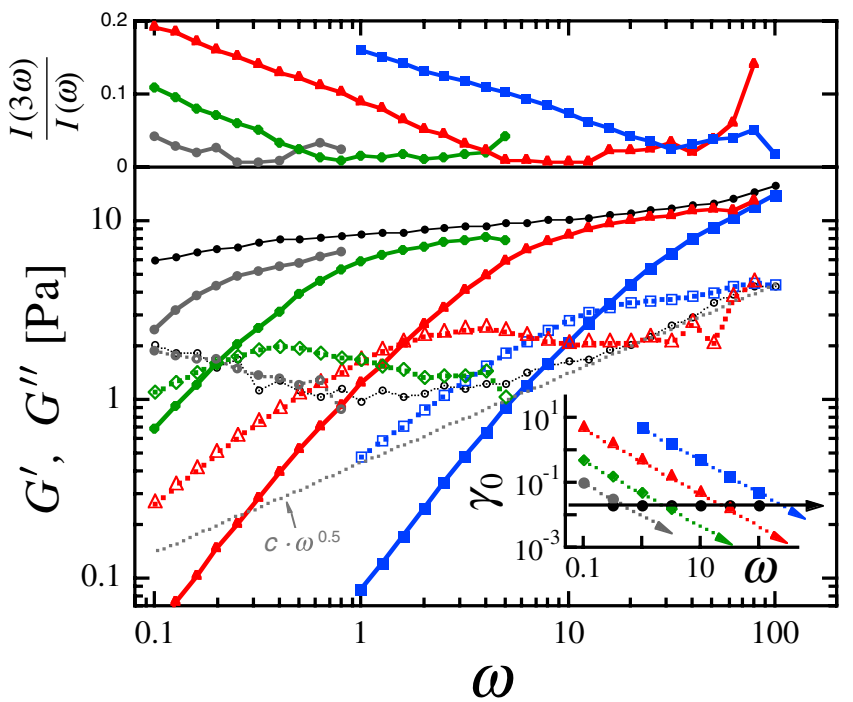

FIG. 2 (color online). Frequency dependent storage $G^{\prime}(\omega)$ (solid symbols) and loss modulus $G^{\prime \prime}(\omega)$ (open symbols), measured in constant rate oscillatory measurements at different strain-rate amplitudes $\dot{\gamma}_{0}=\gamma_{0} \omega ; \dot{\gamma}_{0}=0.01 \mathrm{~s}^{-1}$ (gray circles), $0.05 \mathrm{~s}^{-1}$ (green diamonds), $0.5 \mathrm{~s}^{-1}$ (red triangles), $5 \mathrm{~s}^{-1}$ (blue squares). The linear oscillatory measurement of $G^{\prime}$ and $G^{\prime \prime}$ are shown by the black circles. Inset: Strain amplitude $\gamma_{0}$ versus $\omega$ corresponding to the constant rate measurements in the main graph. Top: Corresponding ratios of the third to the first harmonic response $I(3 \omega) / I(\omega)$ as a function of $\omega$.
[Fig. 1(b)] are performed at constant $\omega$, probing the viscoelastic response at a fixed time scale $2 \pi / \omega$. However, the probe frequency might not be the only important time scale. Instead, as the strain rate becomes large, it can itself drive the slow structural relaxation process at the time scale of the imposed strain rate. For oscillatory measurements, the natural scale of strain rate is its amplitude $\dot{\gamma}_{0}=$ $\gamma_{0} \omega$. Thus, if a structural relaxation process is present, its time scale $\tau\left(\dot{\gamma}_{0}\right)$ should depend on the applied strain-rate amplitude,

$$
\frac{1}{\tau\left(\dot{\gamma}_{0}\right)} \approx \frac{1}{\tau_{0}}+K \dot{\gamma}_{0}{ }^{\nu}
$$

However, in traditional strain or frequency dependent rheological measurements, the amplitude of the strain rate varies for each data point for both frequency and straindependent measurements. This makes it difficult to discern the effects of strain rate on the time scale $\tau\left(\dot{\gamma}_{0}\right)$ of a structural relaxation process.

We circumvent this problem by performing frequency dependent rheological measurements at constant strainrate amplitude $\dot{\gamma}_{0}$. This "constant rate frequency sweep" is achieved by applying a strain amplitude that is inversely proportional to the oscillation frequency, as shown in the inset of Fig. 2. We perform a series of such measurements with $\dot{\gamma}_{0}$ ranging from 0.01 to $5 \mathrm{~s}^{-1}$, shown in the main graph of Fig. 2. For comparison, the linear viscoelastic measurement (see Fig. 1) performed at a constant strain amplitude (circles) is also included.

The viscoelastic response depends strongly on the strainrate amplitude. With increasing $\dot{\gamma}_{0}$, the entire frequencydependent response is shifted towards higher $\omega$, as expected from Eq. (1). Remarkably, however, the general shape of the frequency-dependent storage and loss moduli is surprisingly insensitive to $\dot{\gamma}_{0}$. At low frequencies the response is liquidlike: $G^{\prime \prime}$ is larger than $G^{\prime}$ and both moduli scale with frequency as a power law, with a ratio of $\approx 2$ between the corresponding exponents. At higher frequencies there is a pronounced peak in the loss modulus, followed by a shallow minimum and a final slow increase at the highest frequencies. The storage modulus rises continuously until it reaches a plateau where it shows only a very weak frequency dependence. The response remains surprisingly harmonic for all our measurements [6] with a ratio of the third to the first harmonic component of the response that is always smaller than $20 \%$; in the relevant frequency range around the peak position this ratio is smaller than $10 \%$, as shown in the top graph of Fig. 2. This justifies our treatment of the data in terms of the viscoelastic moduli $G^{\prime}$ and $G^{\prime \prime}$.

To highlight the striking similarities in the shape of the viscoelastic response, we rescale the moduli in both magnitude and frequency, using $G_{\text {scaled }}{ }^{*}(\omega)=G^{*}\left(\omega / b\left(\dot{\gamma}_{0}\right)\right) /$ $a\left(\dot{\gamma}_{0}\right)$, where $a\left(\dot{\gamma}_{0}\right)$ and $b\left(\dot{\gamma}_{0}\right)$ are the scaling factors for the magnitude and frequency, respectively. All the data can be 
superimposed onto a single master curve, as shown in Fig. 3. The scaling factors are plotted in the inset to Fig. 3 as a function of $\dot{\gamma}_{0}$. The value of $a\left(\dot{\gamma}_{0}\right)$ varies by less than a factor of 3 over the entire range of $\dot{\gamma}_{0}$ accessed, whereas the frequency scaling factor $b\left(\dot{\gamma}_{0}\right)$ depends strongly on $\dot{\gamma}_{0}$. At large strain rates we observe a powerlaw dependence, $b\left(\dot{\gamma}_{0}\right) \propto \dot{\gamma}_{0}^{\nu}$, with $\nu \approx 0.9$; at low strain rates it asymptotically approaches unity, which suggests that in this regime the imposed strain rate is sufficiently low that it no longer impacts the viscoelastic behavior. The rate dependence of the scaling factors is consistent with the behavior expected from Eq. (1), with $b\left(\dot{\gamma}_{0}\right)=\tau_{0} / \tau\left(\dot{\gamma}_{0}\right)$.

The observed scaling behavior is a direct consequence of a structural relaxation process, which leads to a decrease in $G^{\prime}(\omega)$ and a concomitant peak in $G^{\prime \prime}(\omega)$ in linear viscoelastic measurements. However, this structural relaxation occurs at too low a frequency to be directly observed in the data in Fig. 1(a); nevertheless the incipient behavior is observed in the linear viscoelasticity at low frequencies and is responsible for the peak observed in $G^{\prime \prime}$ in the nonlinear behavior.

The structural relaxation time decreases as $\dot{\gamma}_{0}$ increases, as expected from Eq. (1). The relaxation peaks in the data shown in Fig. 3 occur when $2 \pi / \omega$ becomes comparable to $\tau\left(\dot{\gamma}_{0}\right)$. To isolate this contribution due to structural relaxation, we must separate the peak from the subsequent rise in the data at higher frequencies. Closer inspection of the data reveals systematic differences between the scaled curves at the highest frequencies. This suggests that a significant

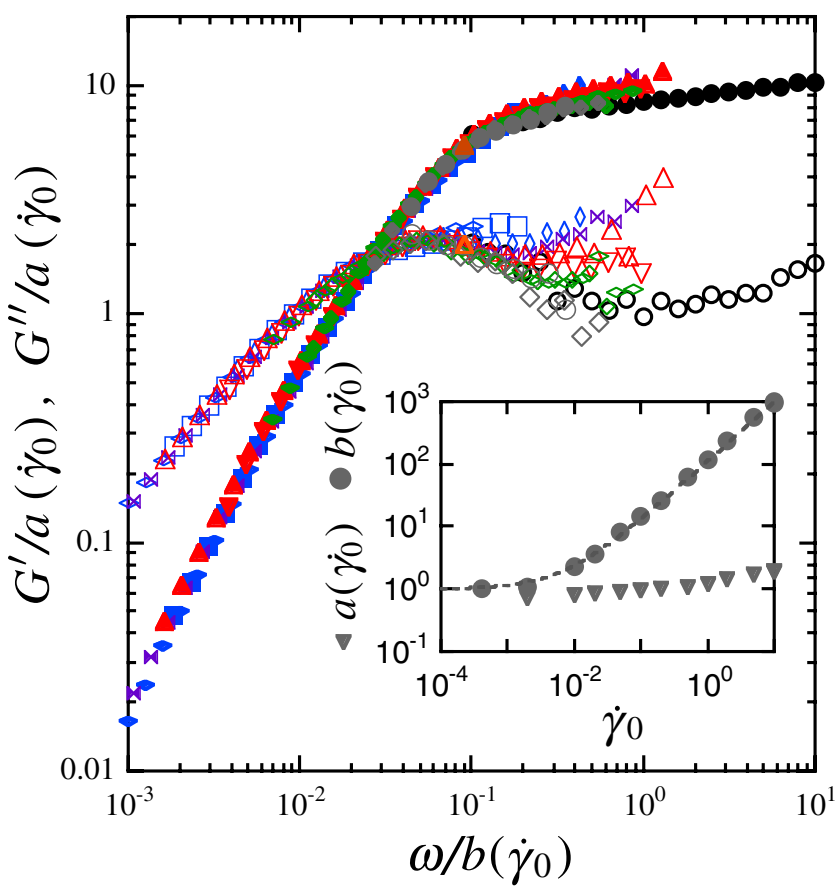

FIG. 3 (color online). Constant rate frequency sweep measurements shifted onto a single master curve. Inset: Corresponding amplitude and frequency shift factors $a\left(\dot{\gamma}_{0}\right)$ and $b\left(\dot{\gamma}_{0}\right)$ versus $\dot{\gamma}_{0}$. contribution to the response does not depend on strain rate at sufficiently high $\omega$. The high frequency viscoelastic response in compressed emulsions exhibits a $\sqrt{\omega}$-dependence, attributed to viscous flow along randomly oriented slip planes [16]; this results in a contribution that scales as $\sqrt{\omega}$. The high frequency linear response of our system shows the same behavior, as shown by the dashed line in Fig. 1(a). We thus subtract a component $c \sqrt{\omega}$ from each of the data sets, where the constant $c$ is determined from a fit to the high $\omega$ response; a single value of $c$ is used for all the data, independent of $\dot{\gamma}$. The resultant loss moduli now exhibit well-defined peaks, as shown in Fig. 4(a); the component subtracted is also shown as the dashed line.

The shapes of the resulting relaxation peaks are remarkably similar at each value of $\dot{\gamma}_{0}$. To highlight this, we scale all the data onto a single master curve by rescaling the frequency, as shown by the uppermost data set in Fig. 4(b); because we have subtracted the $\sqrt{\omega}$ component, we do not need to scale the amplitude. The scaling of the data suggests that the shape of the relaxation spectrum remains unchanged as the strain-rate amplitude is varied. This should provide important new insight about the nature of the relaxation and the distribution of time scales that make up the peak.

The observed scaling suggests that $G^{*}$ can be expressed as a function of frequency and strain amplitude:

$$
G^{*}\left(\omega, \gamma_{0}\right) \approx G_{R}^{*}\left(\omega / b\left(\gamma_{0} \omega\right)\right)+G_{\mathrm{HF}}^{*}(\omega),
$$

where $G_{R}^{*}(\omega)$ describes the functional form of the linear viscoelastic behavior due to the structural relaxation process, and $G_{\mathrm{HF}}^{*}(\omega)$ describes high frequency contributions that are independent of $\gamma_{0}$; caution must be used for $\omega<$ $\omega_{\max }$, where the slowest relaxation must dominate [17].

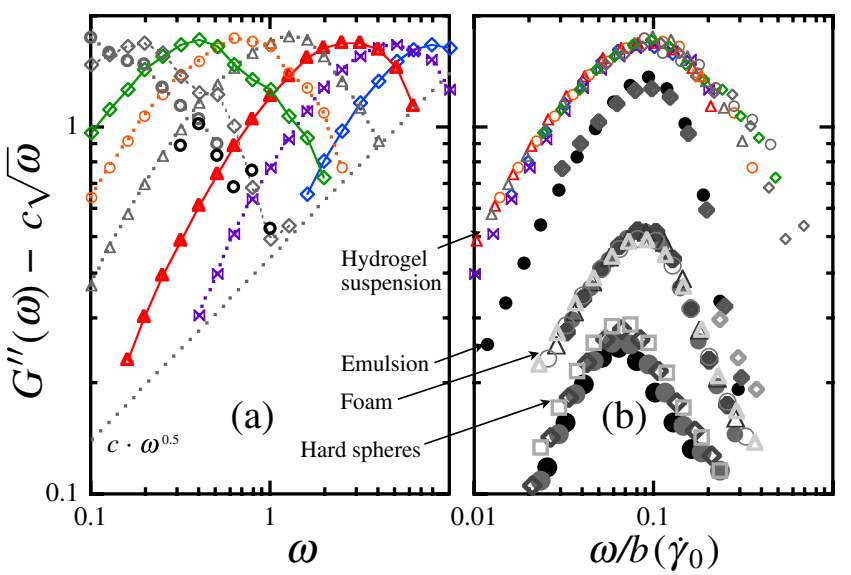

FIG. 4 (color online). (a) Loss modulus $G^{\prime \prime}$ for a series of constant strain-rate oscillatory measurements with a high frequency contribution $c \sqrt{\omega}$ subtracted (dotted line). (b) The uppermost curve shows the same data shifted onto a single master curve. Additional master curves shown: 80 vol \% oil in water emulsion, aqueous foam (Gillette Foamy), PMMA hard-sphere suspension at $\phi \approx 0.58$. Each data set has been scaled for clarity. 
This expression for $G^{*}$ reflects both the linear and the incipient nonlinear response of the system. For low enough values of strain, Eq. (2) describes the complex modulus in the linear viscoelastic regime, with $b\left(\dot{\gamma}_{0}\right) \approx 1$. At larger strains, the structural relaxation shifts towards higher frequencies, scaling as $b\left(\dot{\gamma}_{0}\right)$. This allows us to establish a direct link between linear and nonlinear rheology through the strain-rate dependence of the structural relaxation time. To further illustrate the physical origin of this link, we provide a schematic movie in the supplemental information [18].

Our results provide an important new measurement technique for probing the rheological properties of soft materials. By maintaining a constant strain-rate amplitude during our measurement, we can isolate the structural relaxation; by varying the amplitude of the strain rate, we force the frequency of this structural relaxation into a regime accessible to the rheometer. This allows us to directly probe this structural relaxation, even if it is at a frequency too low to be easily accessible. By scaling the data, we highlight the low-frequency contributions of the structural relaxation. A wide variety of soft materials exhibit such a low-frequency structural relaxation; thus, this method should be very broadly applicable. To illustrate this generality, we perform similar experiments on several representative samples of soft materials, and isolate the structural relaxation by scaling the peak data onto master curves. Similar behavior is observed for all samples as shown in Fig. 4(b), where we plot master curves obtained for a compressed emulsion (80\% silicon oil drops of $\approx 50 \mu \mathrm{m}$ diam. in water, stabilized by $10 \mathrm{~m} M$ SDS), a foam (Gillette Foamy), and a hard-sphere colloidal suspension (PMMA in cycloheptylbromide/decalin). In all cases, good scaling is observed; moreover, differences in shape are also apparent, reflecting different contributions to the relaxation spectrum.

The scaling employed here is reminiscent of an approach that has been successfully employed for polymer melts. Rheological measurements of $G^{\prime}(\omega)$ and $G^{\prime \prime}(\omega)$ for different temperatures are shifted onto a single master curve that reflects the viscoelastic behavior in a dramatically extended range of frequencies. This technique is called time-temperature superposition [19,20]. By analogy, we call our approach strain-rate frequency superposition. With SRFS, we can obtain detailed information on the strain-rate dependence of the slow relaxation process. Our picture combines linear and nonlinear oscillatory rheology into a unified picture, thereby accounting for the unusual behavior typically observed in many soft materials. Our experimental results show that nonlinear viscoelastic measurements contain useful information on the slow relaxation dynamics of these systems. Their yielding behavior directly probes the structural relaxation process itself, shifted towards higher frequencies by an applied strain rate. Physically, this suggests that this structural relaxation, driven by an imposed strain rate, results in the same response as the equilibrium structural relaxation at a much lower frequency. This is a surprising result, as for the steady shear case theory [12] suggests a rate-dependent change of the high frequency flank of the relaxation; for the systems studied we do not observe this in our oscillatory measurements. By exploiting this behavior, SRFS isolates the shape of the structural relaxation, even if it is too slow to be accessed with conventional oscillatory rheology. Thus, SRFS will provide new insight into the physical mechanisms that govern the viscoelastic response of a wide range of soft materials.

This work was supported by the NSF (No. DMR0507208, No. DMR-0602684, No. CHE-0134969), the Harvard MRSEC (No. DMR-0213805), the Hans Werthén, the Wenner-Gren, and the Knut, and Alice Wallenberg Foundations.

[1] T. G. Mason and D. A. Weitz, Phys. Rev. Lett. 75, 2770 (1995).

[2] M. Cloitre, R. Borrega, and L. Leibler, Phys. Rev. Lett. 85, 4819 (2000)

[3] T. G. Mason, J. Bibette, and D. A. Weitz, Phys. Rev. Lett. 75, 2051 (1995).

[4] S. Cohen-Addad, H. Hoballah, and R. Höhler, Phys. Rev. E 57, 6897 (1998).

[5] C. Daniel, I. W. Hamley, M. Wilhelm, and W. Mingvanish, Rheol. Acta 40, 39 (2001).

[6] K. Hyun, S.H. Kim, K.H. Ahn, and S. J. Lee, J. NonNewtonian Fluid Mech. 107, 51 (2002).

[7] P. Sollich, F. Lequeux, P. Hébraud, and M. E. Cates, Phys. Rev. Lett. 78, 2020 (1997).

[8] C. G. Robertson and X. Wang, Phys. Rev. Lett. 95, 075703 (2005).

[9] K. Miyazaki, H. M. Wyss, D. A. Weitz, and D. R. Reichman, Europhys. Lett. 75, 915 (2006).

[10] R. Yamamoto and A. Onuki, Phys. Rev. E 58, 3515 (1998).

[11] L. Berthier and J.-L. Barrat, J. Chem. Phys. 116, 6228 (2002).

[12] M. Fuchs and M.E. Cates, Phys. Rev. Lett. 89, 248304 (2002); Faraday Discuss. 123, 267 (2003).

[13] K. Miyazaki and D. R. Reichman, Phys. Rev. E 66, 050501(R) (2002); K. Miyazaki, D. R. Reichman, and R. Yamamoto, Phys. Rev. E 70, 011501 (2004).

[14] Z. Hu and X. Xia, Adv. Mater. 16, 305 (2004).

[15] X. Xia and Z. Hu, Langmuir 20, 2094 (2004).

[16] A. J. Liu, S. Ramaswamy, T. G. Mason, H. Gang, and D. A. Weitz, Phys. Rev. Lett. 76, 3017 (1996).

[17] H. M. Wyss et al. (to be published).

[18] See EPAPS Document No. E-PRLTAO-98-008724 for a schematic movie and supplemental information. For more information on EPAPS, see http://www.aip.org/pubservs/ epaps.html.

[19] J. Heijboer, Kolloid Z. 148, 36 (1956).

[20] J.D. Ferry, Viscoelastic Properties of Polymers (Wiley, New York, 1980), 3rd ed. 